the significance was only maintained in men $\geq 40$ years.

This study demonstrates a correlation between plasma lipids and ED and suggests an etiologic link. As plasma cholesterol and LDL cholesterol can be normalized through diet and exercise, the authors recommend that these factors be measured regularly, particularly in older individuals. This might then help to reduce the risk of ED and the other complications associated with hyperlipidemia.

Rebecca Doherty

Original article Nikoobakht et al. (2005) The relationship between lipid profile and erectile dysfunction. Int J Impot Res 17: $523-526$

\section{Reducing the gynecomastia and breast pain associated with bicalutamide therapy}

For men who have undergone radical prostatectomy for prostate cancer, adjuvant bicalutamide has been shown to provide survival and quality-of-life benefits. The majority of men receiving bicalutamide experience the adverse effects of gynecomastia and/or breast pain, however. This prospective, multicenter, Italian study directly compared the efficacy of radiotherapy and tamoxifen in reducing such symptoms, and found greater efficacy for tamoxifen.

The study enrolled 102 men receiving adjuvant bicalutamide $150 \mathrm{mg}$ daily, after radical prostatectomy for nonmetastatic prostate cancer. The men were randomly assigned to one of three groups: bicalutamide only ( $n=33$ ); bicalutamide plus tamoxifen $10 \mathrm{mg}$ for 24 weeks $(n=34)$; and bicalutamide plus a single 12 Gy dose of radiotherapy $(n=35)$. Men in the bicalutamide-only group who experienced moderate or severe symptoms were randomly reassigned to one of the other regimens. All were followed up for at least 12 months.

In the bicalutamide-only group, $67 \%$ of men had gynecomastia and $58 \%$ had breast pain, compared with $8 \%$ and $6 \%$, respectively, of those receiving tamoxifen and $34 \%$ and $30 \%$ of those receiving radiotherapy. All differences between the two groups were statistically significant. Men reassigned to tamoxifen showed a greater reduction in symptoms than those reassigned to radiotherapy; at 9 months, only 1 of 11 patients reassigned to tamoxifen still had gynecomastia, and 2 of 10 still had breast pain, compared with 6 of 11 men reassigned to radiotherapy who still had gynecomastia and 7 of 9 who still had breast pain. Longer-term trials are needed to assess the safety of tamoxifen.

Caroline Barranco

Original article Di Lorenzo G et al. (2005) Gynecomastia and breast pain induced by adjuvant therapy with bicalutamide after radical prostatectomy in patients with prostate cancer: the role of tamoxifen and radiotherapy. J Urol 174: 2197-2203

\section{A $2 \mathrm{~cm}$ excision margin is unnecessary for oncologic control in penile carcinoma}

The conventional treatment for penile carcinoma has traditionally been aggressive surgery, with a $2 \mathrm{~cm}$ macroscopic excision margin proposed to ensure adequate clearance of the tumor. However, such radical surgery can lead to erectile and voiding dysfunction and have an adverse psychological impact on the patient. A recent move towards conservative organsparing techniques has led to improvements in cosmetic and functional outcomes, but the excision margins required to achieve local control are still unknown.

Minhas et al. in the UK set out to determine the optimum excision margin based on their experience of conservative surgery for penile carcinoma. A total of 51 patients with biopsyproven squamous cell carcinoma of the penis underwent conservative surgery following clinical and radiological staging.

Perioperative analysis identified three patients $(6 \%)$ with tumor involvement at the excision margins; these patients underwent further surgery to achieve clearance. Histopathologic analysis of the resected specimens showed that $48 \%$ of the excision margins were within $10 \mathrm{~mm}$, and $90 \%$ were within $20 \mathrm{~mm}$, of the tumor edge.

The median follow-up was 26 months, during which time two patients (4\%) underwent partial penectomy for tumor recurrence. This recurrence rate is in accordance with other reports; however, a longer follow-up period is required as delayed local recurrences have been reported.

Tamsin Osborne

Original article Minhas S et al. (2005) What surgical resection margins are required to achieve oncological control in men with primary penile cancer? BJU Int 96: 1040-1043 